\title{
Gate-defined wires in twisted bilayer graphene: From electrical detection of intervalley coherence to internally engineered Majorana modes
}

\author{
Alex Thomson $\odot,{ }^{1,2,3,4, *}$ Ina M. Sorensen $\odot,{ }^{1,2}$ Stevan Nadj-Perge, ${ }^{2,5}$ and Jason Alicea $\odot^{1,2,3}$ \\ ${ }^{1}$ Department of Physics, California Institute of Technology, Pasadena, California 91125, USA \\ ${ }^{2}$ Institute for Quantum Information and Matter, California Institute of Technology, Pasadena, California 91125, USA \\ ${ }^{3}$ Walter Burke Institute for Theoretical Physics, California Institute of Technology, Pasadena, California 91125, USA \\ ${ }^{4}$ Department of Physics, University of California, Davis, California 95616, USA \\ ${ }^{5}$ T. J. Watson Laboratory of Applied Physics, California Institute of Technology, Pasadena, California 91125, USA
}

(Received 22 June 2021; revised 3 November 2021; accepted 10 January 2022; published 9 February 2022)

\begin{abstract}
Twisted bilayer graphene (TBG) realizes a highly tunable, strongly interacting system featuring superconductivity and various correlated insulating states. We establish gate-defined wires in TBG with proximity-induced spin-orbit coupling as (i) a tool for revealing the nature of correlated insulators and (ii) a platform for Majoranabased topological qubits. We show that the band structure of a gate-defined wire immersed in an intervalley coherent correlated insulator inherits electrically detectable fingerprints of symmetry breaking native to the latter. Surrounding the wire by a superconducting TBG region on one side and an intervalley coherent correlated insulator on the other further enables the formation of Majorana zero modes-possibly even at zero magnetic field depending on the precise symmetry-breaking order present. Our proposal not only introduces a highly gate-tunable topological qubit medium relying on internally generated proximity effects but can also shed light on the Cooper-pairing mechanism in TBG.
\end{abstract}

DOI: 10.1103/PhysRevB.105.L081405

\section{INTRODUCTION}

Twisted bilayer graphene (TBG) has emerged as a strikingly versatile platform for correlated phenomena [1-4]. Near the magic twist angle of $\sim 1^{\circ}$, moiré periodicity and interlayer tunneling conspire to generate energetically isolated flat bands that, when partially filled, allow interactions to dominate [5]. To date, experiments have resolved correlation-driven insulators at flat-band fillings of $v=0, \pm 1, \pm 2$, and \pm 3 electrons per moiré unit cell ( $v= \pm 4$ represents full filling/depletion) [1,6-17], reflecting symmetry-breaking electronic instabilities $[18,19]$ whose precise nature remains a largely open question. Superconductivity is additionally often observed adjacent to $v= \pm 2[2,6,8,10-13,15,16]$ and in some samples extends to broader fillings [8]. Crucially, the phase diagram is not only rich but also exquisitely tunable: Due to the giant moiré lattice constant $a_{M} \sim 10 \mathrm{~nm}$, altering the electron density by a modest value of $\sim 10^{12} \mathrm{~cm}^{-2}$ suffices to sweep the system across metallic, band-insulating, superconducting, and correlatedinsulator phases.

Here, we address two ostensibly very different key questions for the field: How can one experimentally reveal the symmetry-breaking order underlying the observed correlated insulators, and can one exploit the richness and tunability of the TBG phase diagram to construct quantum devices for technological applications? To this end, we theoretically explore gate-defined wires in TBG supported by a transition metal dichalcogenide (TMD), e.g., $\mathrm{WSe}_{2}$. Figure 1(a)

*thomson@caltech.edu sketches the architecture, which features a global back gate and a pair of top gates that enable independent tuning of the density in the central wire region and the flanking areas. Recent experiments studied related structures in the context of gate-defined TBG Josephson junctions [20,21]. In our case, the TMD substrate serves to impart appreciable spin-orbit coupling (SOC) - which plays a pivotal role throughout this paper - to the graphene sheets, as seen in many experiments [12,22-35]. Notably, Ref. [12] established that TBG on $\mathrm{WSe}_{2}$ continues to display correlated insulators and superconductivity (the latter over a very broad twist-angle window). Our essential idea is that the electronic properties of the gatedefined wire depend sensitively on the TBG phases realized in its vicinity via internal proximity effects, reflecting leakage of the wire wave functions into the surrounding regions and can thus be tailored by electrostatically controlling the flat-band filling on either side.

When immersed within a given correlated insulator, the band structure of the wire inherits perturbations that reflect the adjacent symmetry-breaking order. We pay special attention to intervalley coherent (IVC) correlated insulators that are leading candidates for the observed insulating phases at $v=0, \pm 2$ [36-39] and have been proposed as parent states of skyrmion-mediated superconductivity [40,41]; their experimental identification is thus particularly important and promises to illuminate the pairing mechanism in TBG. In an IVC state, electrons spontaneously develop coherent intervalley tunneling, thereby breaking translation symmetry on the microscopic graphene (as opposed to moiré) lattice scale [36]. We show that such ultrashort-scale modulations facilitate generation of bandgaps for the wire that would otherwise 


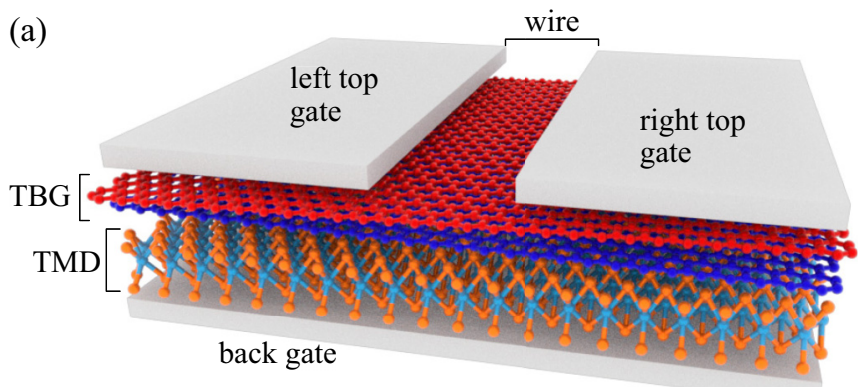

(b)

(c)

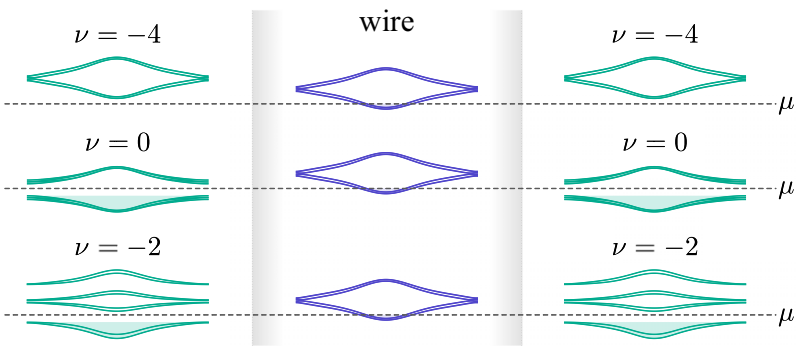

FIG. 1. (a) Gate-defined twisted bilayer graphene (TBG) wire architecture, not to scale. (b)-(d) Schematic flat-band occupations when the wire borders (b) trivial insulators at $v=-4$ and correlated intervalley coherent (IVC) insulators at (c) $v=0$ and (d) $v=-2$

be forbidden - in turn enabling detection of IVC order via large-scale conductance measurements.

The presence of IVC order, if indeed confirmed experimentally, further facilitates engineering Majorana zero modes that are widely coveted for fault-tolerant quantum computing $[42,43]$. Majorana zero modes arise at the endpoints of an odd-channel wire gapped via Cooper pairing [44]. The wellstudied proximitized-nanowire recipe realizes the requisite odd-channel regime through an interplay between Zeeman splitting and SOC that allows gap formation via the proximity effect with a conventional superconductor [45,46]; in gatedefined TBG wires, valley degeneracies must be removed as well in a manner conducive to pairing, posing a nontrivial challenge. The wire bandgaps facilitated by proximate IVC order provide precisely the degeneracy lifting needed to open such an odd-channel regime. Gating one side of the wire into a superconducting phase can then stabilize Majorana zero modes, eschewing the need for external superconducting proximity effects almost universally employed in engineered Majorana platforms. Remarkably, gate-defined TBG wires can potentially harbor Majorana modes even at zero magnetic field depending on details of the IVC order parameter.

\section{TRIVIAL WIRE}

We first examine a gate-defined wire surrounded on both sides by trivial $v=-4$ band insulators that do not spontaneously break any symmetries (similar results of course hold for $v=+4$ ). In the wire region, the chemical potential resides near the flat-band bottom centered around the $\gamma$ point of the moiré Brillouin zone; see Fig. 1(b). Guided by symmetry, we derive a minimal model for the lowest wire subband. The TMD substrate breaks SU(2) spin-rotation symmetry as well as $C_{2}$ symmetry $\left(180^{\circ}\right.$ rotations about the out-of-plane axis) and generates both Ising- and Rashba-type SOC in TBG with respective strengths $\lambda_{I}$ and $\lambda_{R}$. Consequently, the wire preserves only electronic time reversal $\mathcal{T}$ and a $U_{\mathrm{v}}(1)$ valley symmetry associated with conservation of $K$ and $K^{\prime}$ valley quantum numbers (see Refs. [5,47,48]). In terms of momentum-space operators $\psi_{k}$ for the wire and Pauli matrices $\tau^{x, y, z}$ and $s^{x, y, z}$ that respectively act on the (implicit) valley and spin degrees of freedom, these symmetries transform the operators as

$$
\mathcal{T}: \psi_{k} \rightarrow i s^{y} \tau^{x} \psi_{-k}, \quad \mathrm{U}_{\mathrm{v}}(1): \psi_{k} \rightarrow e^{i \phi \tau^{z}} \psi_{k},
$$

where $\phi$ is an arbitrary phase, and $\tau^{z}= \pm 1$ correspond to valleys $K$ and $K^{\prime}$.

We consider the following $\mathcal{T}$ - and $\mathrm{U}_{\mathrm{v}}(1)$-invariant wire Hamiltonian:

$$
H_{0}=\int_{k} \psi_{k}^{\dagger}\left(\frac{k^{2}}{2 m}-\mu+c_{1} k \tau^{z}+k \boldsymbol{\alpha}_{1} \cdot \mathbf{s}+\tau^{z} \boldsymbol{\alpha}_{2} \cdot \mathbf{s}\right) \psi_{k} .
$$

Here, $m$ is the effective mass, $\mu$ is the chemical potential of the wire, $c_{1}$ is a valley-orbit coupling, and $\boldsymbol{\alpha}_{1,2}$ arise from SOC. Figure 2(a) sketches the wire band structure obtained from $H_{0}$. Without SOC (dashed lines), the bands for the two valleys are split by valley-orbit coupling $c_{1}$ but retain twofold spin degeneracy. Resurrecting SOC (solid lines) lifts the spin degeneracy; importantly, the remaining band crossings in the spectrum are protected so long as $\mathrm{U}_{\mathrm{v}}(1)$ is preserved. To emphasize this point, Fig. 2(b) plots the band structure in the presence of a Zeeman term $H_{\mathrm{Z}}=\frac{1}{2} g \mu_{\mathrm{B}} \int_{k} \psi_{k}^{\dagger}(\mathbf{B} \cdot \mathbf{s}) \psi_{k}$ arising from an in-plane magnetic field $\mathbf{B}$ ( $g$ is the electron $g$ factor and $\mu_{\mathrm{B}}$ is the Bohr magneton). Broken time reversal merely shifts the crossings to finite momentum.

\section{A. Wire immersed in $v=0$ IVC order}

Suppose that the wire is instead surrounded by correlated insulators emerging at charge neutrality, i.e., $v=0$ [Fig. 1(c)]. Consider first the case without SOC. There, noninteracting bulk TBG band structure exhibits massless Dirac cones that underpin semimetallicity at $v=0$. Hartree-Fock treatments for pristine TBG, by contrast, predict that Coulomb interactions stabilize an insulating ground state at $v=0$ with IVC order [36-38] (see also Ref. [49]). We will discuss spin-singlet and triplet IVC states-respectively denoted sIVC and tIVC in Figs. 2(c)-2(f) - which are energetically competitive and differentiated by the short-range part of the Coulomb interaction and/or electron-phonon coupling [36]; both also appear compatible with existing measurements [8].

Continuing with the spin-orbit-free problem, spin-singlet IVC order spontaneously breaks time-reversal symmetry $\mathcal{T}$ and $U_{\mathrm{v}}(1)$ but preserves $\mathrm{SU}(2)$ spin rotations as well as an antiunitary operation $\tilde{\mathcal{T}}$ that flips the valley degree of freedom [36]. The last symmetry satisfies $\tilde{\mathcal{T}}^{2}=-1$ and thus, when present, guarantees Kramers degeneracy. When acting on our wire fermions, $\tilde{\mathcal{T}}$ sends $\psi_{k} \rightarrow i \tau^{y} \psi_{-k}$. Resurrecting SOC generically breaks $\tilde{\mathcal{T}}$ symmetry, as can be seen by its nontrivial action on the $\boldsymbol{\alpha}_{1,2}$ terms in Eq. (2). An alternative antiunitary symmetry nevertheless persists:

$$
\mathcal{T}_{\text {sIVC }}: \psi_{k} \rightarrow i s^{y} \tau^{y} \psi_{-k},
$$




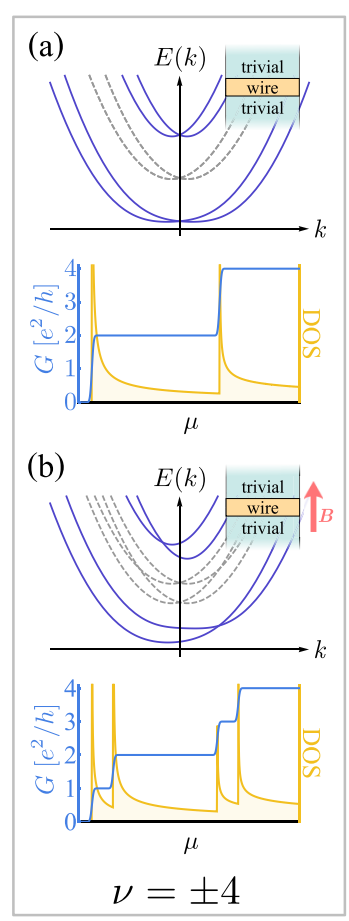

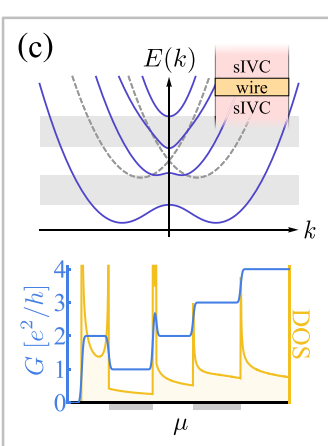

(d)
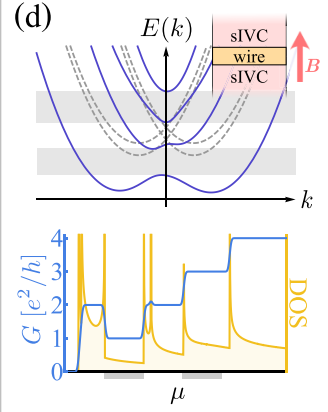

$\nu=0$ (e)

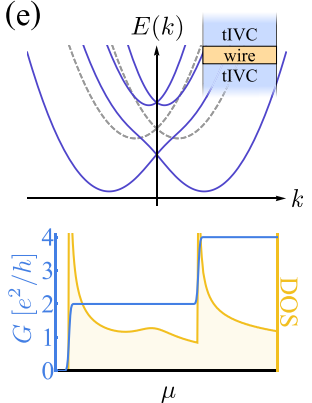

(f)

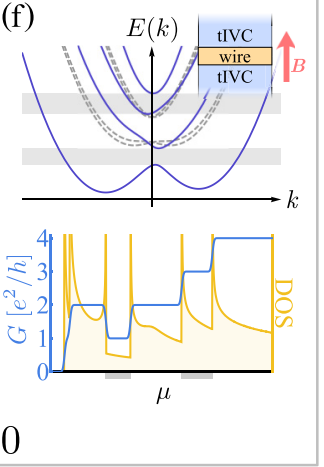

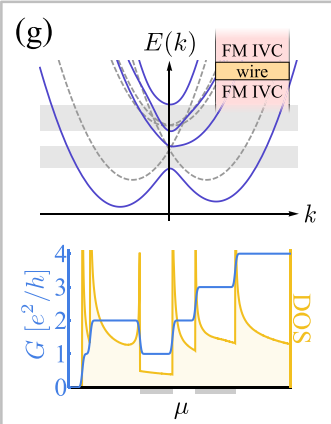

(h)
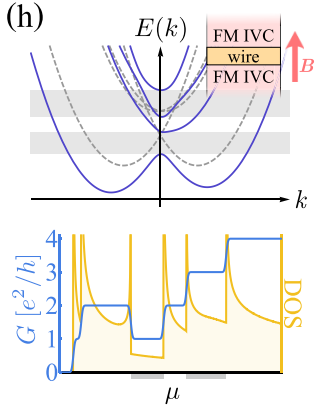

$\nu= \pm 2$

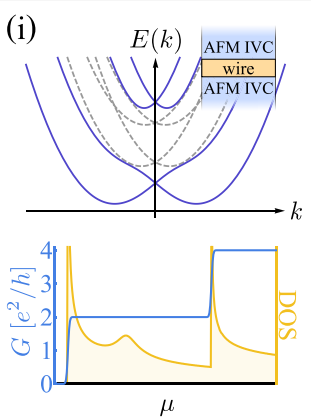

(j)
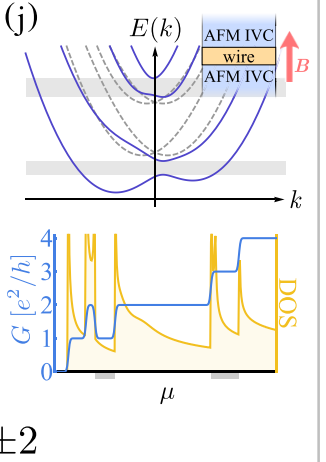

FIG. 2. Band structure, conductance $G$, and density of states for a gate-defined twisted bilayer graphene (TBG) wire immersed in (a) and (b) trivial band insulators and (c)-(j) intervalley coherent (IVC) orders. Insets: Top view of wire and proximate phases. All plots include spin-orbit coupling (SOC) except dashed-line band structures. The upper and lower halves respectively correspond to zero and nonzero in-plane magnetic fields. Proximate IVC order facilitates bandgaps (shaded rectangles) that manifest as conductance dips and associated density of states features within chemical potential windows indicated by gray bars on the $\mu$ axes. The energy window (vertical axis) shown in each top panel is equal to the chemical potential interval (horizontal axis) plotted in the corresponding bottom panel.

corresponding to $\tilde{\mathcal{T}}$ followed by a spin rotation, which indeed leaves Eq. (2) (and the singlet IVC order parameter characterizing the insulating regions) invariant. Notice that $\mathcal{T}_{\text {sIVC }}^{2}=+1$-implying the demise of Kramers degeneracy with $S O C$. Accordingly, the wire band structure in the presence of proximate singlet IVC order [Fig. 2(c)] maintains $k \leftrightarrow$ $-k$ symmetry but generically features no band crossings. Inplane magnetic fields modify the bandgaps and inject $k \leftrightarrow-k$ asymmetry as Fig. 2(d) illustrates.

Without SOC, spin-triplet IVC order spontaneously breaks $\mathrm{SU}(2)$ spin symmetry and $\mathrm{U}_{\mathrm{v}}(1)$ yet preserves both $\mathcal{T}$ and $\tilde{\mathcal{T}}$. Reviving SOC once again breaks $\tilde{\mathcal{T}}$, but unlike the singlet IVC case, we cannot append a spin rotation to obtain a proper symmetry because triplet IVC order breaks spin SU(2). The system then preserves only the familiar electronic time-reversal symmetry $\mathcal{T}$-which satisfies $\mathcal{T}^{2}=-1$ and underpins Kramers degeneracy-implying that proximity to triplet IVC order preserves the crossings in the band structure at $k=0$ [Fig. 2(e)], like the trivial wire case. Contrary to the latter problem, however, the loss of valley conservation from triplet IVC order allows in-plane magnetic fields to eliminate these band crossings; see Fig. 2(f).

\section{B. Wire immersed in $v= \pm 2$ IVC order}

Next, we immerse the wire within a $v= \pm 2$ correlated insulator [Fig. 1(d)]. The commonly observed insulating states at these fillings have also been predicted to display IVC order [36-38]. Insulating IVC states at $v=-2(+2)$ can arise upon completely depleting (filling) two of the fourfold-degenerate flat bands, and then gapping the remaining active carriers via spontaneous intervalley hybridization. We consider in detail two candidate phases that, without SOC, correspond to (i) a ferromagnetic (FM) IVC state with active carriers spin polarized in the out-of-plane $\left(s^{z}\right)$ direction and (ii) an antiferromagnetic (AFM) state with active carriers consisting of $s^{z}=+1$ electrons from one valley and $s^{z}=-1$ electrons from the other. The former state may be relevant to Ref. [50]which reported FM and an anomalous Hall effect at $v=$ 2 in TBG on $\mathrm{WSe}_{2}$ - whereas $v= \pm 2$ insulators observed elsewhere appear compatible with the latter, as argued in Ref. [51].

In the spin-orbit-free problem, both IVC orders spontaneously violate $\mathrm{SU}(2)$ spin symmetry and $\mathrm{U}_{\mathrm{v}}(1)$. The FM IVC state also breaks $\mathcal{T}$ but preserves $\tilde{\mathcal{T}}$; conversely, the AFM IVC state preserves $\mathcal{T}$ but violates $\tilde{\mathcal{T}}$. Turning on SOC breaks $\tilde{\mathcal{T}}$ for the FM IVC state, and (just like the $v=0$ triplet IVC) one cannot append a spin rotation to obtain a modified symmetry because the order parameter breaks spin SU(2). Hence, the wire preserves no symmetries when proximate to FM IVC order and only $\mathcal{T}$ in the AFM IVC case. Figures 2(g)-2(j) present the wire band structures resulting from proximate $\mathrm{FM}$ and AFM IVC order, both with zero (g) and (i) and nonzero (h) and (j) in-plane magnetic fields. The band structures resemble those generated by $v=0$ singlet and triplet IVC order, respectively, though FM IVC order breaks $k \leftrightarrow-k$ symmetry in the band structure even at zero field due to the absence of $\mathcal{T}_{\text {sIVC }}$ symmetry. 
TABLE I. Wire Hamiltonian terms for the trivial wire (first row) and perturbations generated by proximate IVC orders (subsequent rows). Couplings labeled by $a$ are generated by IVC order in the absence of SOC, whereas $\beta$ terms can be viewed as additional IVC orderparameter components generated due to SOC, akin to the spin-orbit-induced admixture of singlet and triplet pairing in inversion-asymmetric superconductors [52]. For $v=0$ triplet and $v= \pm 2$ IVC orders, we assumed that, without SOC, the spins orient in the out-of-plane ( $\pm s^{z}$ ) direction.

\begin{tabular}{lcl}
\hline \hline Proximate order & Wire symmetries & \multicolumn{1}{c}{ Wire perturbations } \\
\hline Trivial wire & $\mathcal{T}^{2}=-1, \mathrm{U}_{\mathrm{v}}(1)$ & $\frac{k^{2}}{2 m}-\mu+c k \tau^{z}+k \boldsymbol{\alpha}_{1} \cdot \mathbf{s}+\tau^{z} \boldsymbol{\alpha}_{2} \cdot \mathbf{s}$ \\
$v=0$ singlet IVC & $\mathcal{T}_{\mathrm{sIVC}}^{2}=+1$ & $a_{1} k \tau^{x}+\tau^{x}\left(\beta_{1}^{x} s^{z}+\beta_{1}^{y} s^{y}\right)+\beta_{2} \tau^{x} s^{z}$ \\
$v=0$ triplet IVC & $\mathcal{T}^{2}=-1$ & $a_{1}^{\prime} k \tau^{x} s^{z}+k \tau^{y}\left(\beta_{1}^{\prime x} s^{x}+\beta_{1}^{y} s^{y}\right)+\beta_{2}^{\prime} \tau^{x}$ \\
$v= \pm 2$ FM IVC & none & $a_{1} k \tau^{x}+a_{1}^{\prime} k \tau^{x} s^{z}+a_{1}^{\prime \prime} k \tau^{z} s^{z}+a_{2}^{\prime \prime} s^{z}+\tau^{x}\left(\beta_{1}^{x} s^{x}+\beta_{1}^{y} s^{y}\right)+\beta_{2} \tau^{x} s^{z}$ \\
$v= \pm 2$ AFM IVC & & $+k \tau^{y}\left(\beta_{1}^{\prime x} s^{x}+\beta_{1}^{y} s^{y}\right)+\beta_{2}^{\prime} \tau^{x}+\beta_{1}^{\prime \prime} k+\beta_{2}^{\prime \prime} \tau^{z}$ \\
\hline \hline
\end{tabular}

Sections A-D in the Supplemental Material [53] complement the preceding symmetry-based analysis-whose qualitative predictions we stress are universal-by deriving the dominant wire-Hamiltonian terms induced by proximate IVC states and SOC at first order in $\lambda_{I, R}$. Table I summarizes the results. The band structures in Fig. 2 were obtained using the corresponding perturbations. Supplemental Material Sec. F [53] also validates qualitative features of the wire band structures using microscopic five-band model simulations.

\section{Experimental IVC detection}

Electrical transport provides a conceptually straightforward diagnostic of the hallmark IVC-mediated wire bandgaps. Blue curves in the lower panels of Fig. 2 sketch the zero-bias, zero-temperature conductance $G$ vs wire chemical potential $\mu$ assuming ballistic transport. Most strikingly, proximate IVC order generates conductance dips (e.g., re-entrant $e^{2} / h$ plateaus) in Figs. 2(c), 2(d), 2(f)-2(h), and 2(j) associated with a bandgap-induced reduction in the number of conducting channels; whether these dips appear at zero or finite magnetic fields additionally reveals the action of time reversal. Notably, the emergence of these $e^{2} / h$ dips for arbitrarily low fields uniquely identifies the state as a $\mathcal{T}$-preserving IVC insulator [Figs. 2(f) and 2(j)]. Conversely, odd conductance plateaus are generically present for $\mathcal{T}$-breaking IVC orders even in the absence of an applied field. Singlet IVC order at $v=0$ can be identified even when the dips widen such that the conductance rises monotonically in $e^{2} / h$ increments, like the trivial case with $B \neq 0$ in Fig. 2(b). The complete lifting of band degeneracy at zero field combined with a vanishing bulk Hall conductance dictated by $\mathcal{T}_{\text {sIVC }}$ symmetry distinguishes singlet IVC order from a state that breaks $\mathcal{T}$ but preserves $\mathrm{U}_{\mathrm{v}}(1)$.

Similar experiments have been conducted in semiconductor-based wires to detect odd-channel helical regimes driven by an interplay between SOC and magnetic fields $[54,55]$. The crucial difference is that odd-channel regimes require inter-valley coherence but, interestingly, do not necessarily require a magnetic field [Figs. 2(c) and 2(g)]. Ballistic conduction is inessential provided the conductance features highlighted above remain visible. The IVC-mediated gaps also qualitatively modify the density of states [lower panels of Figs. 2(c), 2(d), 2(f)-2(i), yellow curves] and can be detected using scanning tunneling microscopy.

\section{Internally engineered Majorana modes}

Shaded rectangles in the Fig. 2 band structures indicate IVC-mediated odd-channel regimes that can be harvested for Majorana modes. Imagine now gating one side of the wire into a superconductor (Fig. 3 insets), which we assume is gapped [21] and pairs time-reversed partners. For accessing topological superconductivity, it suffices to consider the proximity-induced wire pairing perturbation:

$$
\delta H_{\mathrm{SC}}=\frac{1}{2} \int_{k}\left[\psi_{k}^{T}\left(\Delta_{1} \tau^{x} s^{y}+\Delta_{2} \tau^{y} s^{x}\right) \psi_{-k}+\text { H.c. }\right],
$$

with $\Delta_{1,2} \in \mathbb{R}$. The first term is a spin singlet, valley triplet, while the second is a spin triplet, valley singlet; both preserve $\mathrm{U}_{\mathrm{v}}(1)$ and $\mathcal{T}$.

Figure 3 illustrates the phase diagram vs $\mu$ and in-plane magnetic field $B$ at fixed $\Delta_{2}=-0.4 \Delta_{1}$ for a wire bordered on the other end by (a) $v=0$ singlet IVC order and (b) $v= \pm 2$ AFM IVC order. Band structure parameters are the same as for the corresponding panels in Fig. 2. The topological phases (labeled "topo") descending from odd-channel regimes host unpaired Majorana zero modes-which we confirm by simulating the wire model on a lattice with open boundaries.
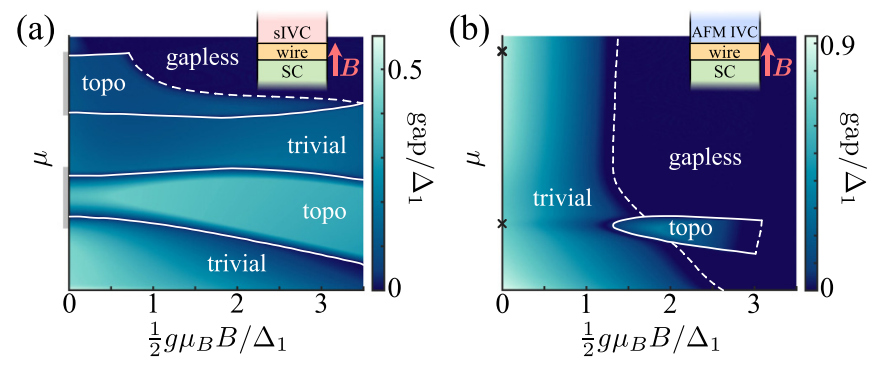

FIG. 3. Phase diagram for a wire bordered on one side by a superconducting TBG region and on the other by (a) $v=0$ singlet IVC order and (b) $v= \pm 2$ AFM IVC order. The color scale shows the wire's excitation gap. 'Topo' indicates topological superconductivity hosting unpaired Majorana zero modes. Grey bars on the $\mu$ axis of (a) correspond to odd-channel regimes highlighted in Fig. 2(c), which give way to Majorana modes even at $B=0$. The crosses on the $\mu$ axis of (b) label the energies at which the Kramers-enforced band crossings occur at $B=0$ in Fig. 2(i). Supplemental Material, Section $E$ presents additional phase diagrams illustrating the dependence on $\Delta_{1,2}[53]$. 
Nonzero $\Delta_{2}$ enables the upper topological phase in Fig. 3(a) and reduces somewhat the critical field for topological superconductivity in Fig. 3(b). Extended gapless regions arise due to suppression of pairing by field-induced $k \leftrightarrow-k$ band asymmetry and, for parameters chosen here, prevent a topological phase from emerging in the upper odd-channel regime in Fig. 2(j). Strikingly, in Fig. 3(a), topological superconductivity extends down to zero magnetic field due to internal $\mathcal{T}$ breaking by the proximate singlet IVC order. Similar behavior is expected from proximate $v= \pm 2$ FM IVC order. In (b), topological superconductivity appears only at $B \neq 0$ since $v= \pm 2$ AFM IVC order preserves $\mathcal{T} ; v=0$ triplet IVC order shares this property and yields a similar phase diagram. The formation of Majorana modes in the latter cases may be assisted by interaction-enhancement of the nominally small $g$ factor of graphene [56] as well as SOC-induced broadening of the field interval over which superconductivity survives in TBG. Moreover, the field orientation comprises a practical tuning knob that can be used to optimize topological superconductivity: The optimal orientation depends on a nonuniversal interplay between the IVC order, SOC parameters, and wire geometry.

\section{OUTLOOK}

Electrical detection of IVC order as envisioned here would not only provide a critical test for skyrmion-mediated superconductivity $[40,41]$ but also lay the groundwork for topological qubit applications. We stress that our proposed experiments extend to other types of IVC states beyond those examined above. Notably, recent Hartree-Fock simulations [39] predict that physically plausible strain levels stabilize a different IVC phase-the intervalley Kekulé spiral (IKS) state-at $v= \pm 2$. Like the AFM IVC state, IKS order preserves $\mathcal{T}$ but violates $\tilde{\mathcal{T}}$ and $\mathrm{U}_{\mathrm{v}}(1)$. The band structure and effective Hamiltonian of a wire immersed within IKS order (supplemented by SOC) thus takes the same generic form as with proximate AFM IVC order. Furthermore, spin-polarized IKS states are proposed at $v= \pm 1, \pm 3$ and appear to be compatible with the experiments of Refs. $[6,17]$. These states accordingly break $\mathcal{T}$ and spin $\mathrm{SU}(2)$ in addition to $\tilde{\mathcal{T}}$ and $\mathrm{U}_{\mathrm{v}}(1)$; the band structure and effective Hamiltonian of a wire proximitized by these orders in turn mimics the FM IVC case. Thus, the IVC diagnostics outlined earlier extend straightforwardly to these cases.

Our proposed gate-defined wire platform offers numerous virtues for Majorana engineering: Ease of gate tunability, internal proximity effects that circumvent interface issues accompanying the merger of disparate materials, real-time control over the arrangement of phases in the device, and amenability to transport and various local probes. Extensions to twisted trilayer graphene [57] are particularly interesting to pursue in future work given that superconductivity persists to higher temperatures [58,59], withstands $\mathrm{O}(10 \mathrm{~T})$ in-plane magnetic fields [60], and appears fully gapped over a range of filling [61]. More generally, we anticipate that gate-defined wires in twisted heterostructures can be broadly employed to diagnose symmetry-breaking order and for quantum devices.

\section{ACKNOWLEDGMENTS}

We are grateful to Cory Dean, Ethan Lake, Cyprian Lewandowski, T. Senthil, and Andrea Young for illuminating discussions. This paper was supported by the Army Research Office under Grant Award No. W911NF17- 1-0323; the U.S. Department of Energy, Office of Science, National Quantum Information Science Research Centers, Quantum Science Center; the National Science Foundation through Grant No. DMR-1723367; an Aker Scholarship; the Caltech Institute for Quantum Information and Matter, an NSF Physics Frontiers Center with support of the Gordon and Betty Moore Foundation through Grant No. GBMF1250; and the Walter Burke Institute for Theoretical Physics at Caltech.

A.T. and I.M.S. contributed equally to this paper.
[1] Y. Cao, V. Fatemi, A. Demir, S. Fang, S. L. Tomarken, J. Y. Luo, J. D. Sanchez-Yamagishi, K. Watanabe, T. Taniguchi, E. Kaxiras, R. C. Ashoori, and P. Jarillo-Herrero, Correlated insulator behaviour at half-filling in magic-angle graphene superlattices, Nature (London) 556, 80 (2018).

[2] Y. Cao, V. Fatemi, S. Fang, K. Watanabe, T. Taniguchi, E. Kaxiras, and P. Jarillo-Herrero, Unconventional superconductivity in magic-angle graphene superlattices, Nature (London) 556, 43 (2018).

[3] L. Balents, C. R. Dean, D. K. Efetov, and A. F. Young, Superconductivity and strong correlations in moiré flat bands, Nat. Phys. 16, 725 (2020).

[4] E. Y. Andrei and A. H. MacDonald, Graphene bilayers with a twist, Nat. Mater. 19, 1265 (2020).

[5] R. Bistritzer and A. H. MacDonald, Moiré bands in twisted double-layer graphene, Proc. Natl. Acad. Sci. USA 108, 12233 (2011).

[6] M. Yankowitz, S. Chen, H. Polshyn, Y. Zhang, K. Watanabe, T. Taniguchi, D. Graf, A. F. Young, and C. R. Dean, Tuning superconductivity in twisted bilayer graphene, Science $\mathbf{3 6 3}$, 1059 (2019).

[7] A. L. Sharpe, E. J. Fox, A. W. Barnard, J. Finney, K. Watanabe, T. Taniguchi, M. A. Kastner, and D. GoldhaberGordon, Emergent ferromagnetism near three-quarters filling in twisted bilayer graphene, Science 365, 605 (2019).

[8] X. Lu, P. Stepanov, W. Yang, M. Xie, M. A. Aamir, I. Das, C. Urgell, K. Watanabe, T. Taniguchi, G. Zhang, A. Bachtold, A. H. MacDonald, and D. K. Efetov, Superconductors, orbital magnets and correlated states in magic-angle bilayer graphene, Nature (London) 574, 653 (2019).

[9] M. Serlin, C. L. Tschirhart, H. Polshyn, Y. Zhang, J. Zhu, K. Watanabe, T. Taniguchi, L. Balents, and A. F. Young, Intrinsic quantized anomalous Hall effect in a moiré heterostructure, Science 367, 900 (2020).

[10] P. Stepanov, I. Das, X. Lu, A. Fahimniya, K. Watanabe, T. Taniguchi, F. H. L. Koppens, J. Lischner, L. Levitov, and D. K. Efetov, Untying the insulating and superconducting orders in magic-angle graphene, Nature (London) 583, 375 (2020). 
[11] Y. Saito, J. Ge, K. Watanabe, T. Taniguchi, and A. F. Young, Independent superconductors and correlated insulators in twisted bilayer graphene, Nat. Phys. 16, 926 (2020).

[12] H. S. Arora, R. Polski, Y. Zhang, A. Thomson, Y. Choi, H. Kim, Z. Lin, I. Z. Wilson, X. Xu, J.-H. Chu, K. Watanabe, T. Taniguchi, J. Alicea, and S. Nadj-Perge, Superconductivity in metallic twisted bilayer graphene stabilized by $\mathrm{WSe}_{2}$, Nature (London) 583, 379 (2020).

[13] A. T. Pierce, Y. Xie, J. M. Park, E. Khalaf, S. H. Lee, Y. Cao, D. E. Parker, P. R. Forrester, S. Chen, K. Watanabe, T. Taniguchi, A. Vishwanath, P. Jarillo-Herrero, and A. Yacoby, Unconventional sequence of correlated Chern insulators in magic-angle twisted bilayer graphene, Nat. Phys. 17, 1210 (2021).

[14] R. Lyu, Z. Tuchfeld, N. Verma, H. Tian, K. Watanabe, T. Taniguchi, C. N. Lau, M. Randeria, and M. Bockrath, Strange metal behavior of the Hall angle in twisted bilayer graphene, Phys. Rev. B 103, 245424 (2020).

[15] Y. Cao, D. Rodan-Legrain, J. M. Park, F. N. Yuan, K. Watanabe, T. Taniguchi, R. M. Fernandes, L. Fu, and P. Jarillo-Herrero, Nematicity and competing orders in superconducting magicangle graphene, Science 372, 264 (2020).

[16] X. Liu, Z. Wang, K. Watanabe, T. Taniguchi, O. Vafek, and J. I. A. Li, Tuning electron correlation in magic-angle twisted bilayer graphene using Coulomb screening, Science 371, 1261 (2021).

[17] P. Stepanov, M. Xie, T. Taniguchi, K. Watanabe, X. Lu, A. H. MacDonald, B. A. Bernevig, and D. K. Efetov, Competing Zero-Field Chern Insulators in Superconducting Twisted Bilayer Graphene, Phys. Rev. Lett. 127, 197701 (2020).

[18] U. Zondiner, A. Rozen, D. Rodan-Legrain, Y. Cao, R. Queiroz, T. Taniguchi, K. Watanabe, Y. Oreg, F. von Oppen, A. Stern, E. Berg, P. Jarillo-Herrero, and S. Ilani, Cascade of phase transitions and Dirac revivals in magic-angle graphene, Nature (London) 582, 203 (2020).

[19] D. Wong, K. P. Nuckolls, M. Oh, B. Lian, Y. Xie, S. Jeon, K. Watanabe, T. Taniguchi, B. A. Bernevig, and A. Yazdani, Cascade of transitions between the correlated electronic states of magic-angle twisted bilayer graphene, Nature (London) $\mathbf{5 8 2}$, 198 (2020).

[20] F. K. de Vries, E. Portoles, G. Zheng, T. Taniguchi, K. Watanabe, T. Ihn, K. Ensslin, and P. Rickhaus, Gate-defined Josephson junctions in magic-angle twisted bilayer graphene, Nat. Nanotechnol. 16, 760 (2020).

[21] D. Rodan-Legrain, Y. Cao, J. M. Park, S. C. de la Barrera, M. T. Randeria, K. Watanabe, T. Taniguchi, and P. Jarillo-Herrero, Highly tunable junctions and nonlocal Josephson effect in magic angle graphene tunneling devices, Nat. Nanotechnol. 16, 769 (2020).

[22] A. Avsar, J. Y. Tan, T. Taychatanapat, J. Balakrishnan, G. K. W. Koon, Y. Yeo, J. Lahiri, A. Carvalho, A. S. Rodin, E. C. T. O'Farrell, G. Eda, A. H. Castro Neto, and B. Ozyilmaz, Spinorbit proximity effect in graphene, Nat. Commun. 5, 4875 (2014).

[23] Z. Wang, D. K. Ki, H. Chen, H. Berger, A. H. MacDonald, and A. F. Morpurgo, Strong interface-induced spin-orbit interaction in graphene on $\mathrm{WS}_{2}$, Nat. Commun. 6, 8339 (2015).

[24] B. Yang, M.-F. Tu, J. Kim, Y. Wu, H. Wang, J. Alicea, R. $\mathrm{Wu}, \mathrm{M}$. Bockrath, and J. Shi, Tunable spin-orbit coupling and symmetry-protected edge states in graphene/ $\mathrm{WS}_{2}, 2 \mathrm{D}$ Mater. 3, 031012 (2016).

[25] Z. Wang, D.-K. Ki, J. Y. Khoo, D. Mauro, H. Berger, L. S. Levitov, and A. F. Morpurgo, Origin and Magnitude of 'Designer' Spin-Orbit Interaction in Graphene on Semiconducting Transition Metal Dichalcogenides, Phys. Rev. X 6, 041020 (2016).

[26] B. Yang, M. Lohmann, D. Barroso, I. Liao, Z. Lin, Y. Liu, L. Bartels, K. Watanabe, T. Taniguchi, and J. Shi, Strong electron-hole symmetric Rashba spin-orbit coupling in graphene/monolayer transition metal dichalcogenide heterostructures, Phys. Rev. B 96, 041409(R) (2017).

[27] T. S. Ghiasi, J. Ingla-Aynes, A. A. Kaverzin, and B. J. van Wees, Large proximity-induced spin lifetime anisotropy in transitionmetal dichalcogenide/graphene heterostructures, Nano Lett. 17, 7528 (2017).

[28] T. Völkl, T. Rockinger, M. Drienovsky, K. Watanabe, T. Taniguchi, D. Weiss, and J. Eroms, Magnetotransport in heterostructures of transition metal dichalcogenides and graphene, Phys. Rev. B 96, 125405 (2017).

[29] S. Zihlmann, A. W. Cummings, J. H. Garcia, M. Kedves, K. Watanabe, T. Taniguchi, C. Schönenberger, and P. Makk, Large spin relaxation anisotropy and valley-Zeeman spin-orbit coupling in $\mathrm{WSe}_{2}$ /graphene/h-BN heterostructures, Phys. Rev. B 97, 075434 (2018).

[30] L. A. Benitez, J. F. Sierra, W. S. Torres, A. Arrighi, F. Bonell, M. V. Costache, and S. O. Valenzuela, Strongly anisotropic spin relaxation in graphene-transition metal dichalcogenide heterostructures at room temperature, Nat. Phys. 14, 303 (2018).

[31] T. Wakamura, F. Reale, P. Palczynski, S. Guéron, C. Mattevi, and H. Bouchiat, Strong Anisotropic Spin-Orbit Interaction Induced in Graphene by Monolayer $\mathrm{WS}_{2}$, Phys. Rev. Lett. 120, 106802 (2018).

[32] J. O. Island, X. Cui, C. Lewandowski, J. Y. Khoo, E. M. Spanton, H. Zhou, D. Rhodes, J. C. Hone, T. Taniguchi, K. Watanabe, L. S. Levitov, M. P. Zaletel, and A. F. Young, Spinorbit-driven band inversion in bilayer graphene by the van der Waals proximity effect, Nature (London) 571, 85 (2019).

[33] D. Wang, S. Che, G. Cao, R. Lyu, K. Watanabe, T. Taniguchi, C. N. Lau, and M. Bockrath, Quantum Hall effect measurement of spin-orbit coupling strengths in ultraclean bilayer graphene/WSe ${ }_{2}$ heterostructures, Nano Lett. 19, 7028 (2019).

[34] T. Wakamura, F. Reale, P. Palczynski, M. Q. Zhao, A. T. C. Johnson, S. Guéron, C. Mattevi, A. Ouerghi, and H. Bouchiat, Spin-orbit interaction induced in graphene by transition metal dichalcogenides, Phys. Rev. B 99, 245402 (2019).

[35] P. Tiwari, S. K. Srivastav, and A. Bid, Electric-Field-Tunable Valley Zeeman Effect in Bilayer Graphene Heterostructures: Realization of the Spin-Orbit Valve Effect, Phys. Rev. Lett. 126, 096801 (2021).

[36] N. Bultinck, E. Khalaf, S. Liu, S. Chatterjee, A. Vishwanath, and M. P. Zaletel, Ground State and Hidden Symmetry of Magic-Angle Graphene at Even Integer Filling, Phys. Rev. X 10, 031034 (2020).

[37] Y. Zhang, K. Jiang, Z. Wang, and F. Zhang, Correlated insulating phases of twisted bilayer graphene at commensurate filling fractions: A Hartree-Fock study, Phys. Rev. B 102, 035136 (2020).

[38] B. Lian, Z.-D. Song, N. Regnault, D. K. Efetov, A. Yazdani, and B. A. Bernevig, Twisted Bilayer graphene IV. Exact insulator 
ground states and phase diagram of twisted bilayer graphene, Phys. Rev. B 103, 205414 (2020).

[39] Y. H. Kwan, G. Wagner, T. Soejima, M. P. Zaletel, S. H. Simon, S. A. Parameswaran, and N. Bultinck, Kekulé spiral order at all nonzero integer fillings in twisted bilayer graphene, Phys. Rev. X 11, 041063 (2021).

[40] E. Khalaf, S. Chatterjee, N. Bultinck, M. P. Zaletel, and A. Vishwanath, Charged skyrmions and topological origin of superconductivity in magic angle graphene, Science 7, 437 (2020).

[41] S. Chatterjee, M. Ippoliti, and M. P. Zaletel, Skyrmion superconductivity: DMRG evidence for a topological route to superconductivity, arXiv:2010.01144.

[42] A. Kitaev, Fault-tolerant quantum computation by anyons, Ann. Phys. 303, 2 (2003).

[43] C. Nayak, S. H. Simon, A. Stern, M. Freedman, and S. Das Sarma, Non-Abelian anyons and topological quantum computation, Rev. Mod. Phys. 80, 1083 (2008).

[44] A. Y. Kitaev, Unpaired Majorana fermions in quantum wires, Sov. Phys.-Uspeki 44, 131 (2001).

[45] R. M. Lutchyn, J. D. Sau, and S. Das Sarma, Majorana Fermions and a Topological Phase Transition in Semiconductor-Superconductor Heterostructures, Phys. Rev. Lett. 105, 077001 (2010).

[46] Y. Oreg, G. Refael, and F. von Oppen, Helical Liquids and Majorana Bound States in Quantum Wires, Phys. Rev. Lett. 105, 177002 (2010).

[47] J. M. B. Lopes dos Santos, N. M. R. Peres, and A. H. Castro Neto, Graphene Bilayer with a Twist: Electronic Structure, Phys. Rev. Lett. 99, 256802 (2007).

[48] H. C. Po, L. Zou, A. Vishwanath, and T. Senthil, Origin of Mott Insulating Behavior and Superconductivity in Twisted Bilayer Graphene, Phys. Rev. X 8, 031089 (2018).

[49] Y. D. Liao, J. Kang, C. N. Breiø, X. Y. Xu, H.-Q. Wu, B. M. Andersen, R. M. Fernandes, and Z. Y. Meng, Correlationinduced insulating topological phases at charge neutrality in twisted bilayer graphene, Phys. Rev. X 11, 011014 (2021).

[50] J.-X. Lin, Y.-H. Zhang, E. Morissette, Z. Wang, S. Liu, D. Rhodes, K. Watanabe, T. Taniguchi, J. Hone, and J. I. A. Li, Science 375, 437 (2022).
[51] E. Lake and T. Senthil, Re-entrant Superconductivity Through a Quantum Lifshitz Transition in Twisted Trilayer Graphene, Phys. Rev. B 104, 174505 (2021).

[52] L. P. Gor'kov and E. I. Rashba, Superconducting 2D System with Lifted Spin Degeneracy: Mixed Singlet-Triplet State, Phys. Rev. Lett. 87, 037004 (2001).

[53] See Supplemental Material at http://link.aps.org/supplemental/ 10.1103/PhysRevB.105.L081405 for details on the symmetry analysis, further studies of the superconducting phases, and wire simulations using the five model.

[54] C. H. L. Quay, T. L. Hughes, J. A. Sulpizio, L. N. Pfeiffer, K. W. Baldwin, K. W. West, D. Goldhaber-Gordon, and R. de Picciotto, Observation of a one-dimensional spin-orbit gap in a quantum wire, Nat. Phys. 6, 336 (2010).

[55] J. Kammhuber, M. C. Cassidy, F. Pei, M. P. Nowak, A. Vuik, O. Gül, D. Car, S. R. Plissard, E. P. A. M. Bakkers, M. Wimmer, and L. P. Kouwenhoven, Conductance through a helical state in an indium antimonide nanowire, Nat. Commun. 8, 478 (2017).

[56] E. M. Stoudenmire, J. Alicea, O. A. Starykh, and M. P. A. Fisher, Interaction effects in topological superconducting wires supporting Majorana fermions, Phys. Rev. B 84, 014503 (2011).

[57] E. Khalaf, A. J. Kruchkov, G. Tarnopolsky, and A. Vishwanath, Magic angle hierarchy in twisted graphene multilayers, Phys. Rev. B 100, 085109 (2019).

[58] J. M. Park, Y. Cao, K. Watanabe, T. Taniguchi, and P. Jarillo-Herrero, Tunable strongly coupled superconductivity in magic-angle twisted trilayer graphene, Nature (London) 590, 249 (2021).

[59] Z. Hao, A. M. Zimmerman, P. Ledwith, E. Khalaf, D. H. Najafabadi, K. Watanabe, T. Taniguchi, A. Vishwanath, and P. Kim, Electric field-tunable superconductivity in alternatingtwist magic-angle trilayer graphene, Science 371, 1133 (2021).

[60] Y. Cao, J. M. Park, K. Watanabe, T. Taniguchi, and P. Jarillo-Herrero, Large Pauli limit violation and reentrant superconductivity in magic-angle twisted trilayer graphene, arXiv:2103.12083.

[61] Y. Cao, J. Min Park, K. Watanabe, T. Taniguchi, and P. JarilloHerrero, Pauli-limit violation and re-entrant superconductivity in moiré graphene, Nature 595, 526 (2021). 